\title{
Discussion of electric engineering automatic control based on the PLC technique
}

\author{
Xiaoqiang Chen ${ }^{1, a}$, Jian Wang ${ }^{2, b}$, Long Li ${ }^{2, c}$, Yantao Liu ${ }^{2, d}$ \\ ${ }^{1}$ Gan Su Yin Guang Chemical Industry Group Co.LTD Enengetic Matenrials Subcompany , Baiyin \\ 730900, China \\ ${ }^{2}$ Gansu Yinguang-Juyin Chemical Limited Company, Baiyin 730900, China

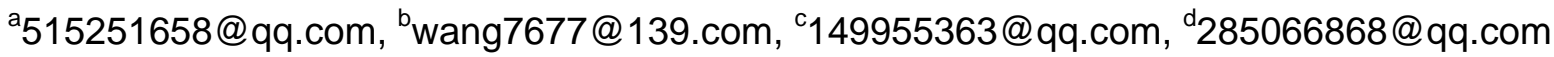

Keywords: PLC technology; electric engineering; automation; control

\begin{abstract}
With the development of information technology, the automation level of electric engineering is continuously increasing. The extensive application of the PLC technique has enhanced the control effect. During the application process of the PLC technique, CPU plays a fundamental role, efficiently improving the control flexibility and reliability. In this paper, the PLC technique is introduced, based on which its application to electric engineering automation is discussed.
\end{abstract}

\section{Introduction}

Influenced by factors such as low technical level and backward devices, traditional electric engineering automatic control system has some defects, resulting in dissatisfying control effects and stagnant automatic level. In order to achieve development, the electric engineering automation industry has adopted the PLC technique in its control system, through which the reliability of the control system is efficiently improved and the automatic level is enhanced. This paper focuses on discussing the application of the PLC technique in electric engineering automatic control.

\section{Overview of the PLC technique}

PLC is the abbreviation of Programmable Logic Controller. It relies on CPU and integrates multiple techniques, including computer techniques, network techniques, communication techniques, etc. In the industrial control system, the PLC technique has a promising application prospect [1]. Since the 1970s, the auto industry has adopted the PLC technique, having achieved favorable application effects. As the PLC techniques keeps on improving, its functions have been enriched and its application scope has been extended to electric automatic control. PLC is an industrial control computer, whose components are basically the same to those of the microcomputer. Generally speaking, the main engine, the I/O extended machine and the peripheral units make up hardware of PLC. (See Fig. 1) In recent years, the flourishing industrial development has led to a continuous increase of the automatic control level in electric engineering. During the application process, the PLC technique has gradually shown its advantages, which have not only extended the storage capacity of electric devices, but also improved the response speed of computers and, in particular, better development of the automatic control of electric engineering. 


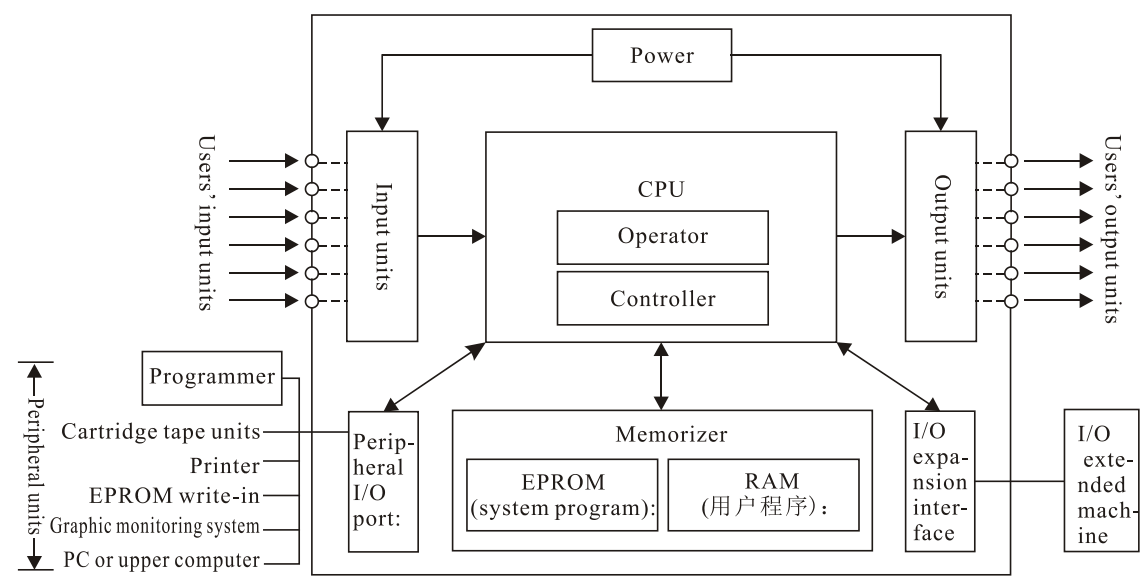

Fig. 1 PLC hardware components

\section{Application of the PLC technique in the electric engineering automatic control}

\section{Sequence control.}

Currently, in the electric auxiliary system, the control methods can be divided into sequence control and switching value control. The automatic technique is employed to reduce the energy consumption of the system and the pollution caused by the system during the practical operation process of the system, and to continuously increase the automatic level during the production and operation process [2]. In the traditional electric auxiliary system, sequence control adopts the relay controller, but fails to achieve the expected control effect. After the adoption of the PLC technique, PLC replaces the relay controller to play a key role, and an increasingly important role in the development of the control system. PID control method is adopted for analog quantity control by PLC. PID features the linear control, and can work out the control deviation based on the set value and the practical output value of PLC. The relationship among the three is $\operatorname{error}(t)=\operatorname{rin}(t)-\operatorname{yout}(t)$, where rin $(\mathrm{t})$ stands for the set value; yout $(\mathrm{t})$ for the practical output value; error $(t)$ for the control deviation. Therefore, the control rules of PID can be deduced: $u(t)=k_{p}\left(\operatorname{error}(t)+\frac{1}{T_{1}} \int_{0}^{1} \operatorname{error}(t) d t+\frac{T_{D} \operatorname{derror}(t)}{d t}\right.$, which can be written into the transfer function, namely $G(s)=\frac{U(s)}{E(s)}=k_{p}\left(1+\frac{1}{T_{1} s}+T_{D} s\right)$. In the function, kp stands for the proportion function; $\mathrm{T} 1$ for the integral time constant; TD for the differentiating time constant. Based on the PID control and the corresponding control procedures (See Fig. 2), independent control technological process is realized during the operation process of the control system, and the production procedures are effectively controlled.

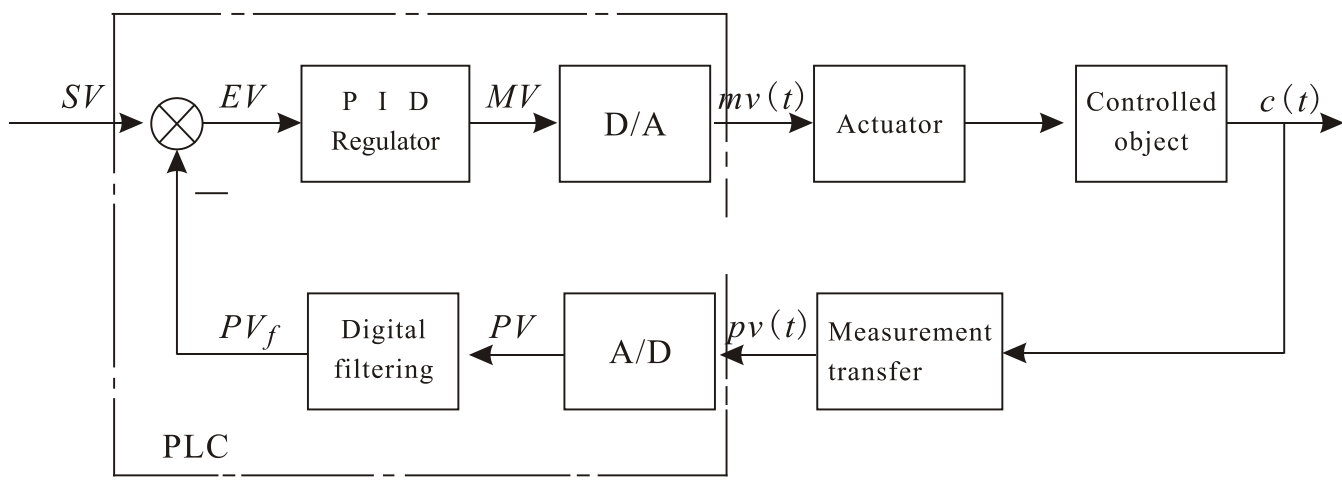

Fig. 2 PID control system structure 


\section{Switching value control.}

In the traditional control system, the magnetic relay is used to control electric devices. During the practical control process, continuous contact faults impairs the reliability of control and the stability of the system. At the same time, the wiring becomes more complex. Once the wiring goes wrong, the short circuit might be easily triggered, influencing the normal operation of the control system. However, after the adoption of the PLC technique, the control system just needs to replace some relays with PLC to achieve effective control. In this way, the stability and reliability of the system operation can be significantly improved, and the system functions can be further improved [3]. In the control system I/O hardware module is used to control the switching value. When the reading is beyond the fixed scope, the corresponding formula, $P=\frac{\operatorname{Max}-\operatorname{Min}}{27648} * I N+\operatorname{Min}$, can be adopted to work out the practical pressure value, P. In the formula, Max stands for the maximum scale value; Min for the minimum scale value; IN for the real-time value tested. The calculation of the formula can efficiently increase the efficiency of control. Besides, after the control system adopts the PLC technique, the number of auxiliary switches used in the system can be significantly reduced. During the control, multiple sets of breakers are concentrated to achieve real-time control. To the power generation system, backup power can enhance the reliability of the system. The specific PLC switching control is shown in Fig. 3.

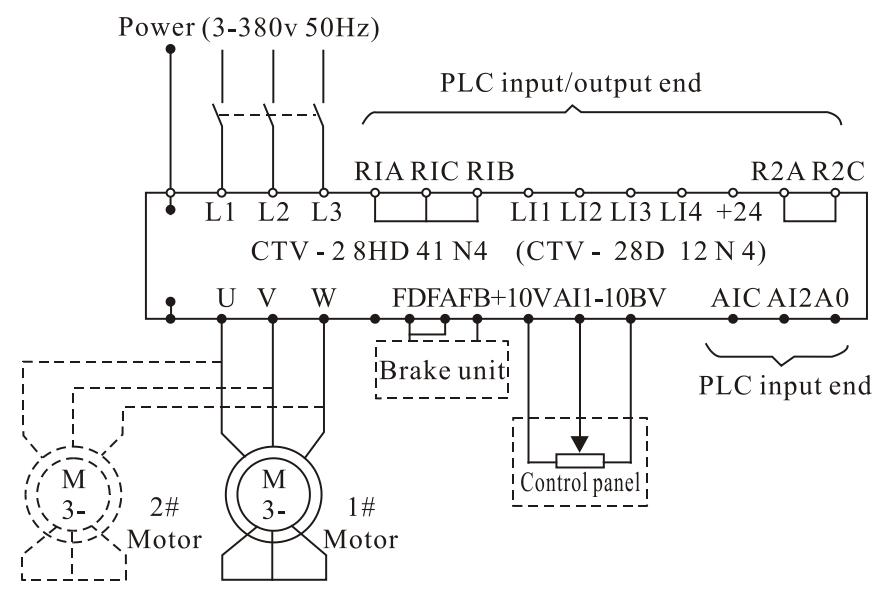

\section{Loop control.}

Fig. 3 Switching value control

In the control system, there are multiple ways to launch the pump motors, but all of them might impair the control stability. After the application of the PLC technique, the pump motors are controlled to efficiently achieve automatic launching. During the control process, the realization of the automatic launching is based on the fuzzy PID algorithm. In the PID fuzzy algorithm, advantages of the fuzzy algorithm and PID algorithm are combined, which can be expressed as the following function, $G s=\frac{K_{M A}}{T_{M} s+1}$. Through the algorithm, the control effect of pump motors can be significantly improved. In fact, PLC has not yet completely replaced the traditional control in the current control system. The combination of the two can efficiently control the pump pumps and ensure the steady operation of pump motors.

\section{Conclusions}

The application of the PLC technique can efficiently improve the stability, reliability and automatic level of the electric engineering automatic control system, and ensure its steady operation. In view of the advantages of the PLC technique, it will have more promising application prospects in the future electric engineering automatic control system. 


\section{References}

[1] XU Qu. Analysis of the application of the PLC technique in the electric automatic control of the chemical industrial equipment [J]. Journal of Xichang College (Natural Science Edition), 2014, (04): 45-47.

[2] YAN Xiaofeng. Application of the PLC technique in the electric equipment automatic control [J]. Technology \& Market, 2015, (08): 182-184.

[3] YU Wei, ZHU Qiuqin \& ZHU Yonghua. Engineering case teaching design for "Electric Control and PLC Technique” [J]. The Guide of Science \& Education, 2013, (04): 131-132. 